\title{
Effect of Tai Chi Versus Aerobic Exercise On Blood Pressure In Prehypertension Patients (TCOBPP): A Study Protocol For A 12-Month Single-Blind Randomized Controlled Trial
}

\section{Xinye Li}

Beijing University of Chinese Medicine

\section{Yonghong Gao}

Beijing University of Chinese Medicine Affiliated Dongzhimen Hospital

Min Wu

Guang'anmen Hospital

Dawei Wei

Beijing University of Chinese Medicine Affiliated Dongzhimen Hospital

\section{Xingjiang Xiong}

Guang'anmen Hospital

\section{Yan Yang}

Beijing University of Chinese Medicine Affiliated Dongzhimen Hospital

\section{Yuchen Jiang}

Guang'anmen Hospital

\section{Xiandu Pan}

Guang'anmen Hospital

\section{Ran Zhao}

Guang'anmen Hospital

\section{Fan Yang}

Guang'anmen Hospital

Jiahao Sun

Guang'anmen Hospital

\section{Shengjie Yang}

Guang'anmen Hospital

\section{Li Tian}

Guang'anmen Hospital

\section{Linqiang Ban}

Guang'anmen Hospital

\section{Xingye Li}




\section{Peifen Chang}

Beijing University of Chinese Medicine Affiliated Dongzhimen Hospital

\section{Yanwei Xing ( $\nabla$ xingyanwei12345@163.com)}

Guang'anmen Hospital

\section{Study protocol}

Keywords: Tai Chi, Aerobic exercise, Prehypertension, Blood pressure

Posted Date: June 29th, 2021

DOl: https://doi.org/10.21203/rs.3.rs-469092/v1

License: (c) (1) This work is licensed under a Creative Commons Attribution 4.0 International License. Read Full License 


\section{Abstract}

Background: Compared with optimal blood pressure (BP), the prehypertension increases the risk of incident hypertension, cardiovascular (CV) events and death. Moderate intensity of regular physical activity can reduce BP. However, aerobic exercise has some limitations. As a safe, low-impact, enjoyable, and inexpensive form of exercise, that requires minimal equipment and space, Tai Chi is expected as a viable alternative to aerobic exercise. The study aimed to assess the effect of Tai Chi intervention program, compared with aerobic exercise, on the BP in prehypertension patients.

Methods: This study is a 12-month, two-center, single-blind, parallel, randomized controlled trial. 340 patients with prehypertension [with a systolic blood pressure (SBP) in the range of $120 \mathrm{mmHg}$ to 139 $\mathrm{mmHg}$ and/or a diastolic blood pressure (DBP) in the range of $80 \mathrm{mmHg}$ to $89 \mathrm{mmHg}$ ] are randomized to one of two intervention groups in a 1:1 ratio: Tai Chi or aerobic exercise. BP monitoring methods of office blood pressure, ambulatory blood pressure monitoring (ABPM) and home blood pressure monitoring (HBPM) are used at the same time to detect BP in multiple dimensions. The primary outcome is the comparison of SBP change from baseline to 12 months in Tai Chi group and SBP change from baseline to 12 months in aerobic exercise group. The secondary endpoints are as following: (1) the comparison of DBP of office blood pressure change from baseline to 12 months between Tai Chi group and aerobic exercise group; (2) the comparison of BP and the variability of BP assessed through ABPM change from baseline to 12 months between Tai Chi group and aerobic exercise group; (3) the comparison of BP assessed through HBPM change from baseline to 12 months between Tai Chi group and aerobic exercise group.

Discussion: This will be the first randomized controlled trial to specifically study the benefits of Tai Chi on the blood pressure control in patients with prehypertension. The successful completion of this study will help to provide evidence for whether Tai Chi is more desirable than aerobic exercise.

Trial Registration Number: Chinese Clinical Trial Registry, ChiCTR1900024368. Registered on 7 July 2019, http://www.chictr.org.cn/edit.aspx?pid=39478\&htm=4

\section{Background}

Prehypertension, defined as blood pressure (BP) in the range of $120-139 / 80-89 \mathrm{mmHg}$, was introduced by the seventh report of the Joint National Committee on Prevention, Detection, Evaluation, and Treatment of High Blood Pressure in 2003 [1]. Prehypertension is highly prevalent and affects $25-50 \%$ of adults worldwide. Compared with optimal BP, the prehypertension increases the risk of incident hypertension, cardiovascular (CV) events and death [2], as it is related to risk factors such as high body mass index, metabolic syndrome, dyslipidemia and impaired glucose metabolism $[3,4]$. Prehypertension increases the risk of incident hypertension, with annual rates ranging from $8-20 \%$ in studies lasting $2-4$ years, and $4-9 \%$ in longer-term studies $[5,6]$. The Guidelines recommend that prehypertension population 
at low-moderate $\mathrm{CV}$ risk should be offered lifestyle advice [7]. Moderate intensity of regular physical activity, as one of the lifestyles, can reduce BP, as well as lower the risk of heart attack and stroke [8].

Aerobic exercise, is recommended as one of the lifestyles that can lower BP [9] and suggested in the guidelines to help both the prevention and treatment of hypertension [7]. However, aerobic exercise has some limitations. The exercise adherence is low and pace and venue also restrict effective training of aerobic exercise. Other useful substitute exercise modes need to be proved effective in reducing BP. As a safe, low-impact, enjoyable, and inexpensive form of exercise, that requires minimal equipment and space, Tai Chi is beneficial to improve exercise adherence and is expected as a viable alternative to aerobic exercise $[10,11]$. Tai Chi guides people to concentrate on slow and fluid movements, covering allround adjustments such as balance, core strength, flexibility and so on [12]. Studies have shown that Tai Chi has a positive effect on patients with cardiovascular diseases (CVD) [13], and it can have a beneficial impact on BP when combined with other lifestyle forms changes [14]. Some studies have shown that after 12 weeks, 9 months or 12 months of intervention, systolic blood pressure (SBP) in the Tai Chi group decreased significantly, suggesting that Tai Chi exercise training can effectively reduce BP in patients with hypertension [15-18].

Therefore, the study aimed to assess the effect of Tai Chi intervention program, compared with aerobic exercise, on the office BP, ambulatory blood pressure monitoring (ABPM) and home blood pressure monitoring (HBPM) in patients with prehypertension. Based on previous studies, Tai Chi was hypothesized to have better effects on lowering BP than aerobic exercise.

\section{Methods /design Study design}

This protocol followed the Standard Protocol Items: Recommendations for Interventional Trials (SPIRIT) reporting guideline (Fig. 1 and additional file 1). The study setting is two hospitals in Beijing, China: Guang'anmen Hospital of China Academy of Chinese Medical Sciences and Dongzhimen Hospital of Beijing University of Chinese Medicine. The TCOBPP study has received approval from the Ethics Committee of two hospitals.

This study is a 12-month, parallel, randomized controlled trial, conducted in two centers. Patients with prehypertension are randomized to one of two intervention groups: 6-month supervised Tai Chi held four times a week, or 6-month supervised aerobic exercise. After the 6-month treatment sessions, participants are encouraged to continue Tai Chi or aerobic exercises until 12 months. All groups will be followed up for 12 months.

A one-week induction period is designed for those who meet the inclusion criteria for prehypertension screening; during this period, participants will receive Tai Chi education and train to exclude individuals who cannot tolerate the exercises and improve study compliance. Outcome measurements are collected at baseline, 3 months, 6 months, and 12 months (Table 1). The staff conducting the BP assessments and 
the statistician are blinded to treatment tasks and groupings. The study flow chart shows an overview of the study procedure (Fig. 2).

Table 1

Sequence of Primary and secondary outcomes measurement during intervention and follow-up

\begin{tabular}{|c|c|c|c|c|}
\hline & Baseline & $\begin{array}{l}\text { Month } \\
3\end{array}$ & $\begin{array}{l}\text { Month } \\
6\end{array}$ & $\begin{array}{l}\text { Month } \\
12\end{array}$ \\
\hline Time (days) & $-7 \sim 0$ & $90 \pm 14$ & $180 \pm 14$ & $360 \pm 14$ \\
\hline \multicolumn{5}{|l|}{ Primary outcome variable } \\
\hline SBP in office blood pressure ${ }^{a}$ & $x$ & & $x$ & $x$ \\
\hline \multicolumn{5}{|l|}{ Secondary outcome variables } \\
\hline DBP in office blood pressure & $x$ & & $x$ & $x$ \\
\hline ABPM $^{b}$ & $x$ & & & $x$ \\
\hline Caloric assessment of $\operatorname{diet}^{C}$ & $x$ & $x$ & $x$ & $x$ \\
\hline HBPM & $x$ & $x$ & $x$ & $x$ \\
\hline IPAQ $^{d}$ & $x$ & $x$ & $x$ & $x$ \\
\hline SF-36 & $x$ & & & $x$ \\
\hline Blood taken & $x$ & & & $x$ \\
\hline Urine taken & $x$ & & & $x$ \\
\hline SCORE system & $x$ & & & $x$ \\
\hline
\end{tabular}

asystolic blood pressure (SBP) in office blood pressure is the primary outcome at 12 months; the other collection times are secondary outcome variables. ${ }^{\mathrm{b}}$ Ambulatory blood pressure monitoring (ABPM) can provide the average blood pressure estimates during the whole monitoring period, and provide average BP during nighttime and daytime respectively, estimate the variability of BP. ${ }^{\mathrm{C}}$ Assess the average daily calorie intake of participant for the nearly week. dUsed to assess the one-week total physical activity including physical activity of occupation, transportation, housework and recreation.

Abbreviations: DBP, diastolic blood pressure; HBPM, home blood pressure monitoring; IPAQ, International Physical Activity Questionnaire; SF-36, Medical Outcome Survey Short-Form 36; SCORE, Systematic COronary Risk Evaluation.

\section{Eligibility Criteria}

Patients who meet the classification of prehypertension [with a SBP in the range of $120 \mathrm{mmHg}$ to 139 $\mathrm{mmHg}$ and/or a diastolic blood pressure (DBP) in the range of $80 \mathrm{mmHg}$ to $89 \mathrm{mmHg}$ ] are eligible to 
participate in this study [1]. Recruitment strategies include the distribution of flyers within the hospital as well as advertisements in print and online media, to ensure adequate enrollment of different study population. Potential participants will be contacted by phone and WeChat to assess whether they meet the basic eligibility criteria for the study. Those who meet the eligibility criteria are invited to come to the hospital for screening, in which case their eligibility criteria are verified. After a complete explanation of the study procedures, each eligible subject who agrees to participate provides informed consent, which is completed by the principal investigator or study coordinator. The study coordinator will inform participants of the schedule of the training sessions, including the date and time.

Participants are considered eligible for this study if they: (1) are aged from 18 to 65 years; (2) fulfill the classification of prehypertension: with a SBP in the range of $120 \mathrm{mmHg}$ to $139 \mathrm{mmHg}$ and/or a DBP in the range of $80 \mathrm{mmHg}$ to $89 \mathrm{mmHg}$ [1]; (3) have no western medicine or traditional Chinese medicine, acupuncture and moxibustion were used to control blood pressure (or the treatment was discontinued for 2 weeks); (4) are willing to be randomized to Tai Chi group or aerobic exercise group; (5) have ability to complete written questionnaires and operate electronic equipment independently; (6) are able to give informed consent. Exclusion criteria are (1) diagnosed secondary hypertension; (2) pregnant and lactating women; (3) non-dominant arm circumference $>50 \mathrm{~cm}$; (4) body mass index (BMI) larger than $40.0 \mathrm{~kg} / \mathrm{m}^{2}$; (5) take benzodiazepines, antipsychotics or oral glucocorticoids (allowed to taken fluoxetine, paroxetine, sertraline, fluvoxamine, citalopram and escitalopram stably within 3 months); (6) diagnosed with diabetes mellitus and coronary heart disease; (7) with chronic kidney disease, with eGFR $<60$ $\mathrm{mL} / \mathrm{min}$; (8) with known autonomic neuropathy (such as Shy-Drager syndrome, orthostatic hypotension, white coat hypertension); (9) alcoholism (male's alcohol intake is more than $25 \mathrm{~g} /$ day or $140 \mathrm{~g} /$ week or female's alcohol intake is more than $15 \mathrm{~g}$ /day or $80 \mathrm{~g} /$ week) ; (10) has played Tai Chi more than once a month in the past six months; (11) plays vigorous sports activities more than three times a week; (12) with musculoskeletal disorder or other disabling diseases lead to the inability to practice Tai Chi or do aerobic exercise; (13) current in clinical trials of other drugs or external therapies.

\section{Randomization and blinding}

Participants, after the induction period, who met all the eligibility criteria and provided written informed consent will be randomly assigned to either Tai Chi or aerobic exercise (control) group, in a 1:1 ratio, with a predicted sample size of 340 patients, 170 in each group. At the same time, the participants are stratified according to SBP of $120-129 \mathrm{mmHg}$ and DBP $<80 \mathrm{mmHg}$, and SBP of $130-139 \mathrm{mmHg}$ or DBP of $80-89 \mathrm{mmHg}$. At each stratum, the proportion of participants in the Tai Chi and aerobic exercise groups is also randomly assigned in a 1:1 ratio. In order to ensure the concealment of allocation, a 24hour central web-based automated randomisation system is adopted for all randomisation processes, using the static random method and the SAS9.4 software PROC PLAN process programming.

When allocation is complete, the outcome assessors who evaluate the effects of the treatments will receive only the participant number, and then interpretation the data under blinded to group allocation.

\section{Sample Size}


The sample size calculation was based on the comparison of the office SBP drop of individuals in the Tai Chi group and the aerobic exercise group. According to the average reduction of SBP in the studies conducted before the start of the trial $[15,19]$, using a conservative estimate, we hypothesized that the SBP in the Tai Chi group would be reduced by $4.5 \mathrm{mmHg}$ more than the aerobic exercise group. Further conservatively assuming an SD of 13.0 of both groups. We estimate a loss of follow-up of $20 \%$ and $80 \%$ power at a two-sided a level of 0.05 . Therefore, 170 participants per group and 340 participants in total is the reasonable sample size of this study. The analyses were performed using PASS version 15.0.

\section{Study intervention}

The maximum waiting time between baseline assessment and interventional therapy is 3 weeks. In order to avoid the influence of seasonal factors on the disease, both Tai Chi and aerobic exercise groups are simultaneously performed. Participants randomly assigned to Tai Chi or aerobic exercise will practice at indoor activity room in or near Guang'anmen Hospital or Dongzhimen Hospital. The number of participants in each class is limited to 20 to ensure the quality of teaching and learning.

\section{Lifestyle intervention}

Common interventions are health education and lifestyle guidance. Participants in both groups receive dietary recommendations for weight control and salt intake. DASH eating plan is the best diet that can effectively reduce BP [20]. During the study intervention, participants are advised to follow the DASH diet, which is rich in fruits, vegetables and low-fat dairy foods and with reduced saturated and total fat.

\section{Tai Chi intervention}

The 24-form Yang-style Tai Chi consists of 24 standard movements. The four Tai Chi instructors each have extensive experience and will explain and demonstrate Tai Chi principles, practice techniques and safety precautions for each movement at the beginning of the study. The instructor will review these principles and techniques, as throughout the study process and always practice with the participants, to timely and effectively identify and correct the incorrect posture or movement. Participants will also be instructed to concentrate and perform traditional Tai Chi breathing, while performing body movements. Moreover, all four instructors completed the required human subject protection training before the beginning of intervention courses.

Each Tai Chi session will last for $60 \mathrm{~min}$, including $10 \mathrm{~min}$ of warm-up exercise, $40 \mathrm{~min}$ of Tai Chi teaching and/or practice, and 10 min of relaxation, and occur 4 times a week. Among them, there are no less than twice centralized sessions per week, and for the rest practice, participants can practice at home and upload videos. The instructor could modify and tailor-make for their Tai Chi exercises according to participants' learning and athletic ability. In the initial eighth-week, the participants learn and practice step by step. In each session, participants practice and learn 3 to 4 movements of Tai Chi. After all of the 24 Tai Chi forms had been learned (weeks 10 and 11), the Tai Chi instructor (experience $>10$ years) will assess the participants. Participants who are able to perform the 24 forms of Tai Chi independently and skillfully will be awarded a qualification certificate, and those, who are unable to, will be screened off the 
study. After completing the 6-month treatment sessions, participants will be asked to continue Tai Chi exercises for up to 12 months. Participants are required to sign in to confirm the accurate attendance records, when they attend the Tai Chi session or practice at home. The standard case report forms are used to record and verify the data collected for class attendance, to confirm accurate attendance recordings. The study staff will monitor the participants by monthly home calls throughout the 12-month follow-up. Throughout the study, all sessions are regularly monitored and fed back to ensure proper instruction.

Aerobic exercise intervention

Participants randomized to aerobic exercise will receive a supervised, group-format aerobic exercise program. The aerobic exercises training protocol for prehypertension treatment consists of four 60minute sessions of moderate intensity exercises per week. The aerobic exercises include climbing stairs, jogging, brisk walking and cycling. Each session includes several parts: 10 minutes of warm-up including low-intensity exercise and dynamic stretching; 40 minutes of organized aerobic training, gradually developing from low intensity to medium intensity; 10 minutes cool-down. The training in the sessions is progressive, and all participants gradually increase the duration and intensity of the exercise. In all sessions, instructors will closely monitor to ensure the comfort and safety of participants and to minimize adverse events. Heart rate will be recorded during each session to monitor the intensity of exercise. During the 1-4 weeks, participants are advised to achieve an individualized heart rate of $55 \%-$ $65 \%$ of estimated maximum heart rate according to their age, and should reach $60 \%-70 \%$ after 4 weeks. The maximum heart rate is estimated as "208 - $0.7 \times$ age"[21]. Participants in aerobic exercise group will be recommended to perform the above exercises no less than 2 times a week, including collective exercises no less than 1 time a week, and the rest 1 time of uploaded videos. A wrist wearable device (HUAWEI band) is used to monitor heart rate. It uses an optical sensor to accurately calculate heart rate through fluctuations in blood flow in the wrist. The data are uploaded to an app, where information is stored for long time. The data collected for session attendance are recorded using standard case report forms. The sessions lasted for 6 months, after which participants are encouraged to continue aerobic exercises until 12 months. The study team will contact the participants by monthly phone to monitor their adherence until the 12-month follow-up evaluation. The sessions lasted for 6 months, after which participants are encouraged to continue aerobic exercises until 12 months. During the study process, all sessions are regularly monitored by the instructors and fed back to ensure correct instruction for the group.

\section{Measurements}

Referring to the BP measurement method in the Guideline [22], the specific measurement method of the study is as follows. A quiet room is set up for measurement of BP. The upper arm medical electronic sphygmomanometer certified by the internationally accepted protocol is used (Omron HBP-1300). The patients are asked to rest, sitting in a chair, for $>10 \mathrm{~min}$, and the first BP measurement is conducted following the rest period. The participant and the researcher should not talk during the rest period or the measurement. The interval of repeated measurements is 1-2 minutes. When the difference between the 
first two measurements is greater than $10 \mathrm{mmHg}$, additional measurements are taken. The average of the last two readings is recorded to estimate the individual's level of BP.

Participants receive 24 h-ambulatory blood pressure monitoring (Welch Allyn ABPM 6100). The 24-hour ABPM is programmed to automatically obtain BP records, with the instrument set to obtain readings every 30 minutes throughout the day and every 1 hour at night. ABPM can provide the average BP estimates during the whole monitoring period, and provide average BP during nighttime and daytime respectively, estimate the variability of $\mathrm{BP}$.

Participants are given a free upper-arm cuff device (Lifesense i5S), which enables automatically stores multiple readings, and educated on its use. Referring to the home BP monitoring measurement in the Guideline [7], measurements are taken in a quiet room after $5 \mathrm{~min}$ of rest, with the participant seated. Participants are instructed to obtain home BP measurements two times in the morning after voiding and before eating or vigorous exercise and two times in the evening, with 1 minute apart between readings. Mean home BP is reliable, using the average of two morning and two evening BP readings for at least three consecutive days in one week minimum in each month (23), at the same time, can being used to estimate the variability of BP.

\section{Outcomes and Follow-up}

At enrollment, the essential information of participants' sex, age, nation, income, education level, professional characteristics, personal history, family history, height, weight, waist circumference, hip circumference, eating habits and caloric intake are collected. Overall assessment of BP is focused on. Outcomes are assessed at baseline, 6 months, and 12 months (at the end of the intervention).

\section{Primary outcome}

The primary outcome is the comparison of SBP of office blood pressure change from baseline to 12 months between Tai Chi group and aerobic exercise group.

\section{Secondary outcomes}

BP monitoring methods of office blood pressure, ABPM and HBPM are used at the same time to detect $\mathrm{BP}$ in multiple dimensions. The secondary endpoints are as following:

(1) the comparison of DBP of office blood pressure change from baseline to 12 months between Tai Chi group and aerobic exercise group; (2) the comparison of BP and the variability of BP assessed through ABPM change from baseline to 12 months between Tai Chi group and aerobic exercise group; (3) the comparison of BP assessed through HBPM change from baseline to 12 months between Tai Chi group and aerobic exercise group.

\section{Monitoring of Adverse Events}

Adverse events must be registered during the study period, which refers to the medical conditions not considered as end points of study. Study participants are monitored for the occurrence of adverse events, 
during each encounter during the study intervention. A study telephone number is provided to the participants to report adverse events throughout the study. All adverse events are recorded in the case report form during the study intervention.

\section{Criteria for study withdrawal}

Individuals who decide to no longer participate in the study or who are lost to follow-up (failure to attend the prescheduled visits or cannot be reached by telephone call) can be withdrawn from the study.

\section{Statistical Analysis}

The data of all participants who completed the follow-up will be analyzed according to the group to which they were originally assigned, regardless of whether having adhered to the treatment and study procedures. Descriptive statistics, such as the mean (SD) or percentage, are used to summarize baseline characteristics and unadjusted study outcome measures, while assessing intergroup equivalence at baseline. At the end of the study, treatment analysis will be performed according to the protocol. Analysis of variance of continuous variables and $\chi^{2}$ (or Fisher exact) test for categorical variables are used to compare baseline demographic descriptions and primary and secondary outcome measures of each group. For continuous variables, the differences between the average variations from baseline values and their respective $95 \%$ Cls will be calculated. If any characteristic is substantially different at baseline, it will be adjusted in a mixed-model regression analysis. To avoid multiple comparisons, differences between groups will be assessed only if the overall effect of treatment is significant. All analyses will be conducted using IBM SPSS (IBM Corp) or Stata (release 13; StataCorp LP). Level of statistical significance will be set at 0.05 .

\section{Discussion}

As far as we know, this is the first randomized controlled trial to specifically study the benefits of Tai Chi on the blood pressure control in patients with prehypertension. Previous study has suggested that prehypertension patients have a twofold to threefold higher risk to develop hypertension than those with normal BP [5]. In a 2017 study, kanegae et al. summarized that the 5-year rates of hypertension in the population with high-normal blood pressure can reach $50 \%$ [24]. Being in the prehypertension period for a long time not only has a significant influence on the cardiovascular system, but also easily develops clinical hypertension.

The Guidelines showed that effective and healthy lifestyle choices can prevent or delay the occurrence of hypertension and reduce CV risk [7]. Physical activity and exercise are the cornerstone of blood pressure control and reducing in some case CV risk, especially before entering the stage of hypertension. Tai Chi, as a meditative exercise, can cause internal functional balance, enhance physiological and psychological functions, so as to promote healing, stress neutralization, and personal harmony [25]. Previous studies have shown that Tai Chi led to effective blood pressure reduction in the short or long term [15-18]. Tai Chi exercise may be an effective method of hypertension, especially for patients who are in the stage of 
prehypertension. It has previously been demonstrated to have a positive effect on blood pressure control. A previous meta-analysis of the effects of Tai Chi on essential hypertension in Chinese adults showed that Tai Chi can reduce the SBP and DBP of hypertensive patients [26]. However, further studies are needed to evaluate the effectiveness of Tai Chi in the treatment of prehypertension. It is worth noting that, in our study, blood pressure monitoring methods of office BP, ABPM and HBPM are used the same time to detect $\mathrm{BP}$ in multiple dimensions and get more comprehensive and multi angle results.

In order to further explore the effect of Tai Chi exercise on prehypertension, this study aims to provide more convincing and detailed information on Tai Chi for better blood pressure control. The successful completion of this study will help to provide evidence for whether Tai Chi is more desirable than aerobic exercise. Through this study, with a large sample and long term of intervention and follow-up (12 months), conducted in two centers, to understand the benefits of Tai Chi in reducing CVD risk factors is of great value to guide the formulation of effective intervention to have an important impact on the public health of prehypertension patients.

\section{Trial Status}

Participant recruitment and randomization began in September 2019. Recruitment is ongoing.

\section{Abbreviations}

ABPM: ambulatory blood pressure monitoring; BMI: body mass index; BP: blood pressure; CV: cardiovascular; CVD: cardiovascular diseases; DBP: diastolic blood pressure; HBPM: home blood pressure monitoring; SBP: systolic blood pressure.

\section{Declarations}

\section{Ethics approval and consent to participate}

The study is conformed to the principles of the Declaration of Helsinki. The study protocol and informed consent document have been approved by the Ethics Committee of Guang'anmen Hospital, China Academy of Chinese Medical Sciences (reference number 2019-086-KY-01). All participants will be informed of the study potential benefits and risks, and then give informed consent prior to study participation. The authors are accountable for all aspects of the work in ensuring that questions related to the accuracy or integrity of any part of the work are appropriately investigated and resolved.

\section{Consent for publication}

Not applicable.

\section{Competing interests}

The authors declare that they have no competing interests. 
Acknowledgments

None.

\section{Funding}

This work was supported by grants from the National Key R\&D Program of China (grants number: 2018YFC1704900 \& 2018YFC1704901).

\section{Availability of data and materials}

Not applicable.

\section{Authors' contributions}

Xinye $L i, P C, M W, D W, X X$ and $Y Y$ drafted the manuscript and will lead, under the guidance of $Y X$, implementation of the study. Xinye $L i, Y X, Y J, X P, R Z, F Y, J S, S Y, L T, L B$, Xingye $L i$ and $Y G$ contributed to the study protocol, including intervention program design, outcome measures collection and data analysis. All authors approved submission of this manuscript.

\section{References}

1. Chobanian AV, Bakris GL, Black HR, et al. Seventh report of the Joint National Committee on Prevention, Detection, Evaluation, and Treatment of High Blood Pressure. Hypertension (Dallas, Tex.: 1979) 2003;42(6):1206-52.

2. Egan BM, Stevens-Fabry S. Prehypertension-prevalence, health risks, and management strategies. Nat Rev Cardiol. 2015;12(5):289-300. Epub 2015 Feb 17.

3. Ganguly SS, Al-Shafaee MA, Bhargava K, Duttagupta KK. Prevalence of prehypertension and associated cardiovascular risk profiles among prediabetic Omani adults. BMC Public Health. 2008;8:108.

4. Grotto I, Grossman E, Huerta M, Sharabi Y. Prevalence of prehypertension and associated cardiovascular risk profiles among young Israeli adults. Hypertension (Dallas, Tex.: 1979) 2006;48(2):254-9.

5. Egan BM, Stevens-Fabry S. Prehypertension-prevalence, health risks, and management strategies. Nat Rev Cardiol. 2015;12(5):289-300.

6. Huang Y, Wang S, Cai X, et al. Prehypertension and incidence of cardiovascular disease: a metaanalysis. BMC Med. 2013;11:177.

7. Williams B, Mancia G, Spiering W, et al. 2018 ESC/ESH Guidelines for the management of arterial hypertension. European heart journal. 2018;39(33):3021-104.

8. Grundy SM, Stone NJ, Bailey AL, et al. 2018 AHA/ACC/AACVPR/AAPA/ABC/ACPM/ADA/AGS/APhA/ASPC/NLA/PCNA Guideline on the 
Management of Blood Cholesterol. A Report of the American College of Cardiology/American Heart Association Task Force on Clinical Practice Guidelines. Circulation 2019;139(25):e1082-e143.

9. Haskell WL, Lee IM, Pate RR, et al. Physical activity and public health: updated recommendation for adults from the American College of Sports Medicine and the American Heart Association. Circulation. 2007;116(9):1081-93.

10. Wayne PM, Kaptchuk TJ. Challenges inherent to t'ai chi research: part I-t'ai chi as a complex multicomponent intervention. J Altern Complement Med. 2008 Jan-Feb;14(1):95-102.

11. Wayne PM, Kaptchuk TJ. Challenges inherent to t'ai chi research: part II-defining the intervention and optimal study design. J Altern Complement Med. 2008 Mar;14(2):191-7.

12. Zheng S, Kim C, Lal S, Meier P, Sibbritt D, Zaslawski C. The Effects of Twelve Weeks of Tai Chi Practice on Anxiety in Stressed But Healthy People Compared to Exercise and Wait-List Groups-A Randomized Controlled Trial. Journal of clinical psychology. 2018;74(1):83-92.

13. Pan L, Yan J, Guo Y, Yan J. Effects of Tai Chi training on exercise capacity and quality of life in patients with chronic heart failure: a meta-analysis. Eur J Heart Fail. 2013;15(3):316-23.

14. Maris SA, Quintanilla D, Taetzsch A, et al. The combined effects of tai chi, resistance training, and diet on physical function and body composition in obese older women. Journal of aging research. 2014;2014:657851.

15. Chan AWK, Chair SY, Lee DTF, et al. Tai Chi exercise is more effective than brisk walking in reducing cardiovascular disease risk factors among adults with hypertension: A randomised controlled trial. International journal of nursing studies. 2018;88:44-52.

16. Ma C, Zhou W, Tang Q, Huang S. The impact of group-based Tai chi on health-status outcomes among community-dwelling older adults with hypertension. Heart lung: the journal of critical care. 2018;47(4):337-44.

17. Sun J, Buys N. Community-Based Mind-Body Meditative Tai Chi Program and Its Effects on Improvement of Blood Pressure, Weight, Renal Function, Serum Lipoprotein, and Quality of Life in Chinese Adults With Hypertension. The American journal of cardiology. 2015;116(7):1076-81.

18. Tsai JC, Wang WH, Chan P, et al. The beneficial effects of Tai Chi Chuan on blood pressure and lipid profile and anxiety status in a randomized controlled trial. Journal of alternative and complementary medicine (New York, N.Y.) 2003;9(5):747 - 54.

19. Wu Y, Johnson BT, Chen S, et al. Tai Ji Quan as antihypertensive lifestyle therapy: A systematic review and meta-analysis. J Sport Health Sci. 2020: S2095-2546(20)30038-7.

20. Schwingshackl L, Chaimani A, Schwedhelm C, et al. Comparative effects of different dietary approaches on blood pressure in hypertensive and pre-hypertensive patients: A systematic review and network meta-analysis. Critical reviews in food science nutrition. 2019;59(16):2674-87.

21. Tanaka H, Monahan KD, Seals DR. Age-predicted maximal heart rate revisited. J Am Coll Cardiol. 2001;37(1):153-6.

22. Whelton PK, Carey RM, Aronow WS, et al. 2017 ACC/AHA/AAPA/ABC/ACPM/AGS/APhA/ASH/ASPC/NMA/PCNA Guideline for the Prevention, 
Detection, Evaluation, and Management of High Blood Pressure in Adults: A Report of the American College of Cardiology/American Heart Association Task Force on Clinical Practice Guidelines. Hypertension (Dallas, Tex.: 1979) 2018;71(6):e13-e115.

23. Bello NA, Schwartz JE, Kronish IM, et al. Number of Measurements Needed to Obtain a Reliable Estimate of Home Blood Pressure: Results From the Improving the Detection of Hypertension Study. Journal of the American Heart Association. 2018;7(20):e008658.

24. Kanegae H, Oikawa T, Kario K. Should Pre-hypertension Be Treated? Curr Hypertens Rep. 2017;19(11):91.

25. Jahnke R, Larkey L, Rogers C, Etnier J, Lin F. A comprehensive review of health benefits of qigong and tai chi. Am J Health Promot: AJHP. 2010;24(6):e1-25.

26. Lian ZY, Yang LL, Bian YY, et al. Effects of Tai chi on adults with essential hypertension in China: A systematic review and meta-analysis. European Journal of Integrative Medicine. 2017;12:153-62.

\section{Figures}




\begin{tabular}{|c|c|c|c|c|c|c|}
\hline \multirow[b]{3}{*}{ TIMEPOINT } & \multicolumn{6}{|c|}{ STUDY PERIOD } \\
\hline & \multirow{2}{*}{$\begin{array}{c}\text { Enrolment } \\
\text { week-1- (-1) }\end{array}$} & \multirow{2}{*}{$\begin{array}{c}\text { Allocation } \\
0\end{array}$} & \multicolumn{3}{|c|}{ Intervention } & \multirow{2}{*}{$\begin{array}{l}\text { Close-out } \\
\text { month } 12\end{array}$} \\
\hline & & & Month 1-5 & Month 6 & month 7-11 & \\
\hline \multicolumn{7}{|l|}{ ENROLMENT: } \\
\hline $\begin{array}{l}\text { Eligibility } \\
\text { screen }\end{array}$ & $X$ & & & & & \\
\hline $\begin{array}{l}\text { Informed } \\
\text { consent }\end{array}$ & $X$ & & & & & \\
\hline Allocation & & $x$ & & & & \\
\hline \multicolumn{7}{|l|}{ INTERVENTIONS: } \\
\hline \multicolumn{7}{|l|}{ Tai Chi group } \\
\hline \multicolumn{7}{|l|}{$\begin{array}{l}\text { Aerobic exercise } \\
\text { group }\end{array}$} \\
\hline \multicolumn{7}{|l|}{ ASSESSMENTS: } \\
\hline $\begin{array}{l}\text { Baseline } \\
\text { characteristics }\end{array}$ & $x$ & & & & & \\
\hline $\begin{array}{l}\text { SBP in office blood } \\
\text { pressure }\end{array}$ & $X$ & & & $x$ & $X$ & $X$ \\
\hline $\begin{array}{l}\text { DBP in office blood } \\
\text { pressure }\end{array}$ & $\mathrm{X}$ & & & $x$ & $x$ & $x$ \\
\hline ABPM & $x$ & & & & & $x$ \\
\hline $\begin{array}{l}\text { Caloric assessment of } \\
\text { diet }\end{array}$ & $x$ & & & & $x$ & $x$ \\
\hline HBPM & $x$ & & & & $x$ & $x$ \\
\hline IPAQ & $x$ & & & & $x$ & $x$ \\
\hline SF-36 & $x$ & & & & & $x$ \\
\hline Blood taken & $x$ & & & & & $x$ \\
\hline Urine taken & $x$ & & & & & $x$ \\
\hline SCORE system & $x$ & & & & & $\mathrm{X}$ \\
\hline Adverse events & & & $x$ & $x$ & $x$ & $x$ \\
\hline
\end{tabular}

Figure 1

SPIRIT figure. Schedule Of enrolment, interventions, and assessments. 


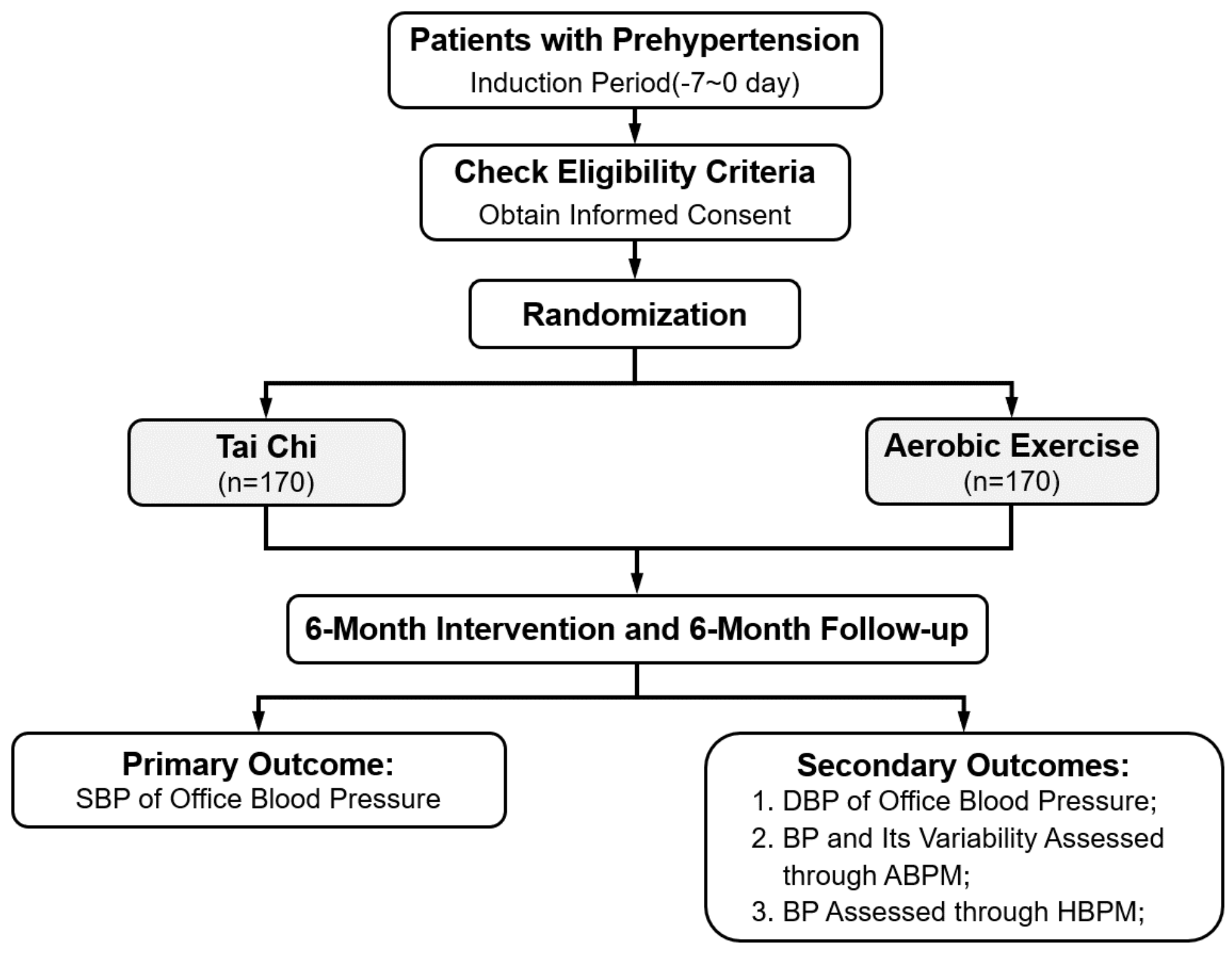

Figure 2

Study flow chart. SBP, systolic blood pressure; DBP, diastolic blood pressure; BP, blood pressure; ABPM, ambulatory blood pressure monitoring; HBPM, home blood pressure monitoring.

\section{Supplementary Files}

This is a list of supplementary files associated with this preprint. Click to download.

- SPIRITChecklist.doc 